\title{
Microbiological and Antibiotic Sensitivity Profile of Postoperative Endophthalmitis in a Tertiary Eye Care Hospital of North India
}

\author{
Richa Ranjan ${ }^{1}$, Manisha Agarwal ${ }^{1}$, Shalini Singh ${ }^{1}{ }^{\otimes}$, Rahul Mayor $^{1}$, Lagan Paul $^{1}$, Arpan Gandhi $^{1}$, Neelam Sapra ${ }^{1}$ \\ ${ }^{1}$ Dr. Shroff's Charity Eye Hospital, New Delhi, India
}

\section{ABSTRACT}

Introduction: We describe the microbiological profile of postoperative endophthalmitis from northern India and analyse the pattern of antibiotic sensitivity which may have changed due to development of resistance secondary to overuse of antimicrobial drugs.

Materials and methods: This is a retrospective study of postoperative endophthalmitis from January 2011 to December 2017 in a tertiary eye hospital of northern India. Any patient developing endophthalmitis within one year of any intraocular procedure was included in the study. According to the severity, treatment with a trial of intravitreal antibiotic injections or core vitrectomy was decided. Ocular samples were collected which were either anterior chamber tap or vitreous biopsy and sent for microbiological examination. The samples were stained with Gram's and KOH stain and cultured on chocolate agar, blood agar, brain heart infusion broth and Sabouraud dextrose agar.

Results: A total of 545 patients of postoperative endophthalmitis were analysed which showed a male predilection (60.5\%) with maximum patients between the age group 50-69 years. 292 patients (53.5\%) were culture negative and 253 patients (46.4\%) were culture positive.

Most common organism identified was Staphylococcus in 73 patients followed by Pseudomonas in 48 patients. Staphylococcus species was most sensitive to vancomycin (97\%) followed by amikacin (91\%) followed by gentamicin and moxifloxacin ( $88 \%$ each). Pseudomonas was the second most common isolate which showed maximum sensitivity to imipenem ( $82 \%$ ) followed by ciprofloxacin( $60 \%)$. Polymicrobial infection was noted in 23 patients. The most common fungal isolate was Aspergillus in 11 patients, followed by Fusarium in 10 patients.

Conclusion: Our study shows that gram positive bacteria are the most common organisms in postoperative endophthalmitis and are most sensitive to vancomycin, followed by gram negative bacteria which shows increased sensitivity with imipenem than commonly used antibiotic, ceftazidime.

Key words: Antibiotic sensitivity profile, Microbiological profile, Postoperative endophthalmitis.

Financial Interest : Nil

Conflict of Interest : Nil

Corresponding Author

Dr. Shalini Singh

Dr. Shroff's Charity Eye Hospital,

Kedar Nath Road, Daryaganj,

New Delhi, India.

E-mail: drshalini15@gmail.com

Contact: +919958927755
Received : 07.11.2020

Accepted : 21.12. 2020

Access this article online

Website: www.nepjol.info/index.php/NEPJOPH

DOI: https://doi.org/10.3126/nepjoph.v13i2.29255

Copyright $\odot 2021$ Nepal Ophthalmic Society ISSN: 2072-6805, E-ISSN: 2091-0320

This work is licensed under a Creative Commons Attribution-NonCommercial-NoDerivatives 4.0 International License (CC BY-NC-ND). 


\section{INTRODUCTION}

Postoperative endophthalmitis is an infective intraocular inflammatory condition caused by microbial organisms (bacteria, fungi or parasites) that infect the eye during the perioperative period (Barry et al., 2006). The identification of the causative infectious agent is essential for treating with therapeutic agents to which it is most sensitive, and to understand the epidemiology and plan its prevention (Jambulingam et al., 2010). For optimal visual outcomes, early diagnosis and intervention with appropriate therapeutic drugs and surgical intervention are mandatory. The most common cause of postoperative endophthalmitis in India, continues to be cataract surgery, (Lalitha et al, 2005; Jambulingam et al, 2010; Sharma et al, 2014; Khanna et al, 2015), as it the most common eye surgery performed.

The incidence of the causative infective organisms may differ from different geographic areas depending on the atmosphere and often camp settings, temperature, humidity etc. On review of literature, various studies have shown different microbiological profile from different geographical areas such as a study from western India showed coagulase negative Staphylococcus as the most common isolate (Kelkar et al., 2016) whereas another study from southern India showed Nocardia as the most common isolate (Lalitha et al., 2005). There is a lacuna in literature for the microbiological profile of postoperative endophthalmitis from northern India with one study showing Aspergillus flavus as the most common isolate and Pseudomonas aeruginosa as the most common bacterial isolate (Gupta et al., 2003). Therefore, we describe the microbiological profile of postoperative endophthalmitis from northern India and analyse the pattern of the antibiotic sensitivity which may have changed due to the development of resistance secondary to overuse of antimicrobial drugs.

\section{MATERIALS AND METHODS}

A retrospective analysis of all the medical records of all the patients diagnosed with post-operative endophthalmitis including in-house and referred from January 2011 to December 2017 in a tertiary eye hospital of northern India were reviewed. Post-operative endophthalmitis was defined as any patient developing endophthalmitis within one year of any intraocular procedures were included in the study. Patients with a history of trauma, history of intraocular surgery more than one year back or no history of intraocular surgery were excluded from the study. Institutional review board and Ethics committee approval was obtained for retrospective data collection and analysis. The research adhered to the tenets of the declaration of Helsinki.

The demographic profile of the patient was recorded including age and gender. All these patients were managed as per the hospital infection control protocol. The patients usually presented within one week of initiation of symptoms. The most common operative procedure leading to endophthalmitis was cataract surgery $(87 \%)$ followed by penetrating keratoplasty $(6 \%)$ followed by trabeculectomy $(5 \%)$. The in-house procedures were operated after initiation of antibiotic eyedrops (moxifloxacin $0.5 \%$ ) one day prior to the surgery, use of $5 \%$ topical povidone-iodine drops into the conjunctival sac for 2 minutes before the start of the surgery, eye patching after the surgery which was removed the next day 
with initiation of post-operative medications. Around $17 \%$ of the procedures included in this study had intraoperative complications like posterior capsular rent, vitreous loss, premature entry, wound leak, bleb leak etc. Since all the post-operative endophthalmitis patients were included from january 2011 to december 2017, the primary surgeon was different in most cases and was informed about the same. However, all the in-house cases were operated under the same aseptic conditions in the operation theatre.

A comprehensive clinical examination which included uncorrected and best corrected visual acuity, slit lamp biomicroscopy, applanation (wherever indicated)/digital tonometry, indirect ophthalmoscopy and ultrasonography was done for all the patients. According to clinical judgement and severity of endophthalmitis, treatment with a trial of intravitreal antibiotic injections or core vitrectomy was decided. In patients treated with a trial of intravitreal antibiotic injections, an anterior chamber (AC) sample was sent for microbiological examination and intravitreal antibiotic injections were given (vancomycin $1 \mathrm{mg}$ in 0.1 $\mathrm{ml}$ normal saline and ceftazidime $2.25 \mathrm{mg}$ in 0.1 $\mathrm{ml}$ normal saline with or without dexamethasone $0.4 \mathrm{mg}$ in $0.1 \mathrm{ml}$ ) after excluding a fungal infection on $\mathrm{KOH}$ preparation. All the patients were started on systemic broad spectrum antibiotics along with topical antibiotics (every 1 hourly), topical prednisolone acetate $1 \%$ (every 4 hourly) and cycloplegic (every 8 hourly). The patients not responding to the above treatment were undertaken for core vitrectomy with intravitreal antibiotic injection and an undiluted vitreous sample was taken prior to the vitrectomy and sent for microbiological examination. A total of 155 patients were primarily treated with intravitreal antibiotic injection whereas 390 patients underwent core vitrectomy surgery. The samples (both AC and vitreous biopsy samples) were stained with grams and $\mathrm{KOH}$ stain for microbiological examination and cultured on 4 medias (blood agar, chocolate agar, brain heart infusion (BHI) broth and Sabouraud dextrose agar (SDA)). All culture medias were evaluated for two weeks before declaring no growth. The patients who showed a response underwent repeat intravitreal injection where the drugs were modified based on the sensitivity report.

Statistical analysis using SPSS version 21.0 was performed which comprised of descriptive analysis and Chi-square test. Chi-square test was applied for proportions. All the patients were followed up for 6 months after resolution of the symptoms.

\section{RESULTS}

A total of 545 postoperative endophthalmitis cases were reviewed from January 2011 to December 2017. Out of this, 330 patients $(60.5 \%)$ were male and $215(39.5 \%)$ were female. The maximum number of patients were in the age group 60-69 years followed by age group 50-59 years. Table 1 shows the demographic profile of the patients.

The microbiological profile of the patients were analysed either by AC tap or vitreous biopsy or both. 171 patients (31.4\%) underwent an aqueous humor sampling, 311 patients $(57.1 \%)$ had a vitreous sampling and 63 patients $(11.5 \%)$ underwent both aqueous and vitreous sampling for microbiological examination.

The samples were stained with Gram and $\mathrm{KOH}$ stains. The sample collected was inoculated on blood agar, chocolate agar, BHI and SDA for culture. Table-2 shows the various organisms 
Table 1 : Demographic profile of patients.

\begin{tabular}{|l|c|c|}
\hline \multicolumn{2}{|c|}{} & No. of patients \\
\hline \multirow{4}{*}{ Gender } & Male & 330 \\
\cline { 2 - 3 } & Female & 215 \\
\cline { 2 - 3 } & $0-9$ & 4 \\
\cline { 2 - 3 } & $10-19$ & 5 \\
\cline { 2 - 3 } & $20-29$ & 17 \\
\cline { 2 - 3 } & $30-39$ & 32 \\
\cline { 2 - 3 } & $40-49$ & 85 \\
\cline { 2 - 3 } & $50-59$ & 153 \\
\cline { 2 - 3 } & $60-69$ & 157 \\
\cline { 2 - 3 } & $70-79$ & 75 \\
\cline { 2 - 3 } & $>80$ & 17 \\
\hline
\end{tabular}

Table 2 : Microbiological analysis of Gram's and KOH staining.

\begin{tabular}{|l|c|l|c|}
\hline GPC & 139 & GPC + Fungal & 3 \\
\hline GPC + Bacilli & 17 & GP diplococci & 2 \\
\hline Bacilli & 7 & Yeast cells & 2 \\
\hline Fungal & 6 & GPC + GNB & 2 \\
\hline GPB & 4 & GPC + GPB & 2 \\
\hline GNB & 4 & GNB + GPB & 1 \\
\hline GP coccobacilli & 3 & GPC + Yeast & 1 \\
\hline
\end{tabular}

(GPC - gram positive cocci, GPB - gram positive bacilli, GNB - gram negative bacilli, GP - gram positive )

identified on microbiological staining with a total of 193 patients showing organism on microbiological staining. Out of the total 545 patients, 292 patients $(53.6 \%)$ were culture negative and 253 patients $(46.4 \%)$ were culture positive. The microorganisms identified were further analysed for antibiotic sensitivity and resistance. The most common organism identified was Staphylococcus in 73 patients followed by Pseudomonas in 48 patients followed by coagulase negative Staphylococcus in 21 patients. Polymicrobial infection was noted in 23 patients. The most common fungal isolate was Aspergillus in 11 patients, followed by Fusarium in 10 patients. Table- 3 shows the various organisms identified on microbiological culture.

The antibiotic sensitivity analysis showed Staphylococcus species to be the most sensitive to vancomycin (97\%) followed by amikacin (91\%) followed by gentamicin and moxifloxacin (88\% each). Pseudomonas was the second most common isolate which showed maximum sensitivity to imipenem (82\%) followed by ciprofloxacin $(60 \%)$. Table 4 shows the antibiotic sensitivity of the various organisms identified. 
Table 3 : Organisms identified on culture.

\begin{tabular}{|l|l|l|}
\hline Staphylococcus - 73 & Streptococcus Viridans - 2 & FUNGI \\
\hline Pseudomonas - 48 & Actinomyces - 2 & Aspergillus - 11 \\
\hline Coagulase negative Staphylococcus - 21 & Enterobacter - 1 & Fusarium - 10 \\
\hline Streptococcus - 13 & GN coccobacilli - 1 & Cephalosporium - 3 \\
\hline Nocardia- 10 & Klebsiella - 1 & Hyaline Fungus - 3 \\
\hline Corynebacterium - 4 & Staphylococcus Citrus - 1 & Candida - 2 \\
\hline Sphingomonas - 4 & Proteus - 1 & Bipolaris species - 1 \\
\hline Atypical Mycobacterium - 3 & Burkholderia species - 1 & Penicillium Fungus - 1 \\
\hline Neisseria - 3 & Moraxella Acinetobacter - 1 & Dematiaceous Fungi - 1 \\
\hline Bacillus - 3 & Mycobacterium - 1 & Cladosporium - 1 \\
\hline GP coccobacilli -3 & Polymicrobial infection - 23 & \\
\hline
\end{tabular}

Table 4 : Antibiotic sensitivity of various organisms.

\begin{tabular}{|l|c|c|c|c|}
\hline & $\begin{array}{c}\text { Staphylococcus } \\
(73)\end{array}$ & $\begin{array}{c}\text { Pseudomonas } \\
(48)\end{array}$ & $\begin{array}{c}\text { Coagulase negative } \\
\text { Staphylococcus }(21)\end{array}$ & $\begin{array}{c}\text { Streptococci } \\
(13)\end{array}$ \\
\hline AK & $63(91 \%)$ & $23(51 \%)$ & $18(100 \%)$ & $7(54 \%)$ \\
\hline CZ & $59(85 \%)$ & $2(0.04 \%)$ & $18(100 \%)$ & $12(92 \%)$ \\
\hline CAZ & $38(55 \%)$ & $26(57 \%)$ & $7(38 \%)$ & $9(69 \%)$ \\
\hline C & $58(84 \%)$ & $11(24 \%)$ & $15(83 \%)$ & $12(92 \%)$ \\
\hline CF & $56(81 \%)$ & $27(60 \%)$ & $14(77 \%)$ & $9(69 \%)$ \\
\hline CT & $3(0.04 \%)$ & $10(22 \%)$ & - & - \\
\hline GF & $49(71 \%)$ & $26(58 \%)$ & $17(94 \%)$ & $9(69 \%)$ \\
\hline GM & $61(88 \%)$ & $18(40 \%)$ & $17(94 \%)$ & $12(92 \%)$ \\
\hline IMP & $1(0.01 \%)$ & $37(82 \%)$ & - & - \\
\hline MF & $61(88 \%)$ & $23(51 \%)$ & $16(88 \%)$ & $11(85 \%)$ \\
\hline OF & $47(68 \%)$ & $24(53 \%)$ & $16(88 \%)$ & $12(92 \%)$ \\
\hline VA & $67(97 \%)$ & $4(0.08 \%)$ & $15(83 \%)$ & $12(92 \%)$ \\
\hline
\end{tabular}

(AK- Amikacin, CZ- Cefazolin, CAZ- Ceftazidime, C- Chloramphenicol, CF- Ciprofloxacin, CT- CoTrimoxazole, GF- Gatifloxacin, GM- Gentamicin, IMP- Imipenem, MF- Moxifloxacin, OF- Ofloxacin, VAVancomycin)

\section{DISCUSSION}

Endophthalmitis is the most dreaded complication following any intraocular surgery. Various studies published show the spectrum of the organisms identified in postoperative endophthalmitis to be different in India in comparison to other parts of the world. There is also a difference in the organisms identified in different geographic regions of India. A study from western India showed coagulase negative staphylococcus as the most common isolate (Kelkar et al., 2006 ) whereas another study from southern India showed nocardia as 
the most common isolates (Lalitha et al., 2005). This could be attributed to various factors such as atmospheric temperature, humidity, primary occupation of that region.

On review of literature we found several reports from southern India, however there was a lacuna in the literature regarding the microbiological profile of postoperative endophthalmitis from North India.

Our study is a retrospective study analysing the microbiological profile and antibiotic sensitivity of postoperative endophthalmitis from January 2011 to December 2017 in a tertiary eye care referral center of northern India. We analysed our data of 545 patients of postoperative endophthalmitis which showed a male predilection $(60.5 \%)$ with maximum patients between the age group 50-69 years, similar to studies published previously and possibly due to maximum number of patients undergoing cataract surgery during this period of age.

Our study showed $35.4 \%$ microscopy positive and $46.4 \%$ culture positive cases which is comparable to the previously published studies (Sharma et al., 2014; Kelkar et al., 2016). In clinically diagnosed endophthalmitis cases in India, the variation in culture positivity varies from $38 \%$ to $44 \%$ (Sharma et al., 2014). Various factors responsible for low culture positivity are low microbial load, delay in processing the sample, prior antibiotic therapy, sequestered microorganisms, etc (Sharma et al., 2014). Therefore for the diagnosis of endophthalmitis, molecular methods such as polymerase chain reaction (PCR) have been recommended. In our study, the samples were processed immediately for microscopy and culture, thus the cause for low yield on culture cannot be attributed to delay in processing.
The Endophthalmitis Virectomy Study (EVS) conducted in the United States reported 94 per cent Gram positive cocci and 6 per cent Gram negative bacilli in postoperative endophthalmitis. A study from northern India showed aspergillus flavus $(67 \%)$ as the most common isolate and pseudomonas aeruginosa $(50 \%)$ as the most common bacterial isolate, however this study analysed occurrence of endophthalmitis post cataract surgery only (Gupta et al., 2003). The most common organism isolated in our study was Staphylococcus (13.4\%) followed by Pseudomonas (8.8\%) and coagulase negative Staphylococcus (3.9\%). Sathpathy G et al., 2017 another study from north India have also reported staphylococcus as the most common isolate. However, studies from south India such as Jambulingam et al., (2010) have reported Staphylococcus, Sharma et al., (2014) and Ambiya et al., (2016) have shown Pseudomonas whereas Kelkar et al., (2016) have reported coagulase negative Staphylococcus as the most common isolate.

Our study showed polymicrobial infection in $4.4 \%$ patients which was similar to that reported by Gupta et al., (2003) and Jambulingam et al., (2010) as $6.5 \%$ and $4 \%$ respectively. The fungal isolates in our study was $6 \%$ which was comparable to that reported by Sathpathy et al., (2017) as $5.3 \%$. However this was markedly reported less as compared to Gupta et al., (2003) who reported $22 \%$ fungal isolates. Table 5 shows a comparative analysis of the results of previous studies and our study.

We also found a few not so common organisms such as Burkholderia species, Moraxella Acinetobacter and Staphylococcus citrus.

Analysis of the antibiotic sensitivity of the microorganism showed $97 \%$ sensitivity of gram 
Table 5 : Comparison of microbiological profile with other published reports.

\begin{tabular}{|c|c|c|c|c|}
\hline & $\begin{array}{c}\text { Number } \\
\text { of eyes }\end{array}$ & $\begin{array}{c}\text { Most common } \\
\text { bacterial isolate }\end{array}$ & $\begin{array}{c}\text { Polymicrobial } \\
\text { infection }\end{array}$ & Fungi \\
\hline $\begin{array}{l}\text { Gupta A et al } \\
\text { (North India study; } \\
\text { 1996-2001) }\end{array}$ & 124 & $\begin{array}{l}\text { 1. Pseudomonas aeruginosa (6) } \\
\text { 2. Staphylococcus aureus (2) } \\
\text { 3. Streptococcus } \beta \text {-hemolyticus (2) }\end{array}$ & $6.5 \%$ & $22 \%$ \\
\hline $\begin{array}{l}\text { Jambulingam } M \text { et al } \\
\text { (South India study; } \\
\text { 2000-2007) }\end{array}$ & 98 & $\begin{array}{l}\text { 1. Staphylococcus (30) } \\
\text { 2. Pseudomonas (19) }\end{array}$ & $4 \%$ & $7.1 \%$ \\
\hline $\begin{array}{l}\text { Sharma S et al } \\
\text { (South India study; } \\
\text { 2006-2009) }\end{array}$ & 46 & $\begin{array}{l}\text { 1. Pseudomonas (4) } \\
\text { 2. Staphylococcus }(2) \\
\text { 3. Streptococcus pneumonia (2) }\end{array}$ & - & $15.2 \%$ \\
\hline $\begin{array}{l}\text { Ambiya V et al } \\
\text { (South India study; } \\
\text { 2010-2014) }\end{array}$ & 100 & $\begin{array}{l}\text { 1. Pseudomonas }(24) \\
\text { 2. Coagulase negative } \\
\text { Staphylococcus }(14) \\
\text { 3. Staphylococcus }(14) \\
\text { Streptococcus }(14) \\
\end{array}$ & $0.04 \%$ & $0.08 \%$ \\
\hline $\begin{array}{l}\text { Kelkar AS et al } \\
\text { (South India study; } \\
\text { 2008-2014) }\end{array}$ & 60 & $\begin{array}{l}\text { 1. } \text { Coagulase negative } \\
\text { Staphylococcus }(8) \\
\text { 2. Staphylococcus }(6) \\
\text { 3. } \text { MRSA coagulase negative (5) }\end{array}$ & - & - \\
\hline $\begin{array}{l}\text { Sathpathy G et al } \\
\text { (North India study; } \\
\text { 2001-2010) }\end{array}$ & 243 & $\begin{array}{l}\text { 1. Staphylococcus }(52) \\
\text { 2. Pseudomonas }(16) \\
\text { 3. Streptococcus }(16)\end{array}$ & - & $5.3 \%$ \\
\hline $\begin{array}{l}\text { Our series } \\
\text { (North India study; } \\
\text { 2011-2017) }\end{array}$ & 545 & $\begin{array}{l}\text { 1. Staphylococcus (73) } \\
\text { 2. Pseudomonas (48) } \\
\text { 3. Coagulase negative } \\
\text { Staphylococcus }(21)\end{array}$ & $4.4 \%$ & $6 \%$ \\
\hline
\end{tabular}

positive cocci to vancomycin. The sensitivity of gram negative organisms to ceftazidime was $57 \%$. This was similar to that reported by Sharma et al., (2014). Gram negative bacilli showed a higher sensitivity $(82 \%)$ to imipenem, similar to results reported by Ambiya V et al., (2016). Hence it is necessary to always do a culture sensitivity test for appropriate management.
Limitation of this study is inclusion of cases from outside of the tertiary eye hospital which can admit confounding factor errors in the study, as cases operated outside have gone through different aseptic conditions of operation theatres with different postoperative management guidelines, also lacking follow up of the referred cases. Whereas strength of the 
study includes large sample size, recent duration of study and confinement to only postoperative endophthalmitis cases.

\section{CONCLUSION}

Our study is one of the largest studies which shows that gram positive bacteria are the most common organisms in postoperative endophthalmitis, followed by gram negative bacteria. Vancomycin was found to be the most sensitive antibiotic to gram positive bacteria however, Pseudomonas being a common gram negative bacteria, shows increased sensitivity with imipenem followed by ciprofloxacin than commonly used antibiotic, ceftazidime. Hence, a microbiology and antibiotic sensitivity is mandatory while the EVS based empiric treatment of postoperative endophthalmitis treatment could be started initially. However, it is necessary to evaluate the clinical response and change the treatment according to the culture sensitivity.

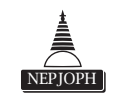

\section{REFERENCES}

Ambiya V, Das T, Sharma S, Chhablani J, Dave V, Jalali S et al (2016). Comparison of clinicomicrobiological profile and treatment outcome of inhouse and referred post cataract surgery endophthalmitis in a tertiary care center in South India. J Ophthalmic Inflamm Infect; 6: 45. doi: 10.1186/s12348-016-0113-0; PMid:27882513

Barry P, Seal DV, Gettinby G, for the ESCRS Endophthalmitis Study Group et al (2006). ESCRS study of prophylaxis of postoperative endophthalmitis after cataract surgery; preliminary report of principal results from a European multicenter study. J Cataract Refract Surg; 32:407-10. doi: 10.1016/j.jcrs.2006.02.021; PMid:16631047

Gupta A, Gupta V, Gupta A, Dogra MR, Pandav SS, Ray P et al (2003). Spectrum and clinical profile of post cataract surgery endophthalmitis in north India. Indian J Ophthalmol; 51:139-45.

Jambulingam M, Parameswaran SK, Lysa S, Selvaraj M, Madhavan HN (2010). A study on the incidence, microbiological analysis and investigations on the source of infection of postoperative infectious endophthalmitis in a tertiary care ophthalmic hospital: An 8 year Study. Indian J Ophthalmol; 58(4): 297-302. doi: 10.4103/03014738.64132; PMid:20534919

Kelkar AS, Kelkar JA, Barve PM, Mulay A, Sharma S, Amoaku W (2016). Post-clear corneal phacoemulsification endophthalmitis: profile and management outcomes at a tertiary eye care center in western India. J Ophthalmic Inflamm Infect; 6: 48. doi: 10.1186/s12348-016-0115-y; PMid:27896783

Khanna RC, Ray VP, Latha M, Cassard SD, Mathai A, Sekhar GC (2015). Risk factors for endophthalmitis following cataract surgery_-Our experience at a tertiary eye care centre in India. Int J Ophthalmol; 8(6):1184-9.

Lalitha P, Rajagopalan J, Prakash K, Ramasamy K, Prajna NV, Srinivasan M (2005). Post cataract endophthalmitis in south India: Incidence and outcome. Ophthalmology; 112:1884-9. doi: 10.1016/j.ophtha.2005.05.020; PMid:16157381

Satpathy G, Nayak N, Wadhwani M, Venkwatesh P, Kumar A, Sharma Y et al (2017). Clinicomicrobiological profile of endophthalmitis: A 10 year experience in a Tertiary Care Center in North India. Indian J Pathol Microbiol; 60:21420. doi: 10.4103/IJPM.IJPM_794_15; PMid:28631638

Sharma S, Padhi TR, Basu S, Kar S, Roy A, Das T (2014). Endophthalmitis patients seen in a tertiary eye care centre in Odisha: A clinicomicrobiological analysis. Indian J Med Res; 139(1):91-8. 